\title{
Gout of the Temporomandibular Joint: Report of a Case
}

\author{
Isaac Nilton Fernandes Oliveira ${ }^{1}$ Renata Caroline Ferreira Gomes ${ }^{1}$ Raiane Rodrigues dos Santos ${ }^{1}$ \\ Thiago de Paula Oliveira ${ }^{1}$ Larissa Loiuse Cândida Pereira ${ }^{1}$ Pietro Mainenti ${ }^{2}$
}

${ }^{1}$ Student, Faculdade de Medicina de Juiz de Fora, Universidade Presidente Antônio Carlos, Juiz de Fora, Minas Gerais, Brazil

2 Professor, PhD, Department of Pathology, Faculdade de Medicina de Juiz de Fora, Universidade Presidente Antônio Carlos, Juiz de Fora, Minas Gerais, Brazil
Address for correspondence Prof. Pietro Mainenti, PhD, Department of Pathology, Faculdade de Medicina de Juiz de Fora, Universidade Presidente Antônio Carlos, Juiz de Fora, Minas Gerais, Brazil (e-mail: pietromainenti@terra.com.br).

Int Arch Otorhinolaryngol 2014;18:316-318.

\begin{abstract}
Keywords

- gout

- temporomandibular joint

- joint diseases

Introduction Gout is an illness characterized by the deposition of monosodium urate crystals in the joints or in soft tissues. The clinical manifestation results from inflammation of limb joints and pain with a rare presentation in the temporomandibular joint (TMJ).

Case Report This study describes a 66-year-old white man with a chief complaint of "occasional pain in the left temporal muscle region." The case disclosed a gout manifestation in the TMJ after physical, radiographic, and ultrasonographic exams, and the patient was referred to proper treatment.

Conclusion Gout manifestation in the TMJ is an unusual presentation, and few reports in the English literature address to the subject. Gout in the TMJ should be included as a differential diagnosis for joint disorders.
\end{abstract}

\section{Introduction}

Gout is an illness characterized by the deposition of monosodium urate crystals in the joints or in soft tissues, ${ }^{1,2}$ leading to acute inflammation with associated pain, impaired movement of the affected joint, and hyperuricemia. ${ }^{3}$ The small joints of the limbs and the elbows and knees are the usually affected sites. ${ }^{3-5}$ The temporomandibular joint (TMJ) is rarely involved. ${ }^{3-5}$ There are currently few reports in the English medical literature relating gout and TMJ. The article aims to report a presentation of gout in the TMJ and discuss the pertinence of the role of physical and complementary exams addressed to the case.

\section{Case Report}

A 66-year-old white man presented for consultation at the Department of Oral and Maxillofacial Surgery, Centro Médico Rio Branco, in May 2011. He reported occasional pain in the left temporal muscle region for 1 year. In this period, the patient used nonsteroidal anti-inflammatory drugs (NSAIDs) with improvement of the symptoms. The longest period of NSAID use lasted a month. At the consultation, the patient complained about a volumetric augmentation in the aforementioned region. There was no history of diseases, although the patient reported hyperuricemia in previous hematologic exams.

Extraoral physical examination showed a volumetric augmentation in the left temporal region, matching the patient report ( - Fig. 1). Massage of the left parotid gland showed no purulent discharge. The patient denied previous trauma in the examined regions.

Due to the suspicion of a gout manifestation, complementary exams were requested. The blood tests showed high glucose (117 mg/dL-reference values: 60 to $99 \mathrm{mg} / \mathrm{dL}$ ), high creatinine $(1.6 \mathrm{mg} / \mathrm{dL}-$ reference values: 0.4 to $1.3 \mathrm{mg} / \mathrm{dL})$, and high uric acid (8.1 mg/dL-reference value: 3.4 to $7.0 \mathrm{mg} /$ dL). Ultrasonography indicated a normal parotid gland and a left hypoechoic palpable area, with well-defined contours measuring $3.7 \times 1.0 \mathrm{~cm}$ (-Fig. 2). According to the received

September 3, 2013

accepted

September 25, 2013

published online

January 7, 2014
DOI http://dx.doi.org/

10.1055/s-0033-1363464. ISSN 1809-9777.
Copyright $(2014$ by Thieme Publicações License terms Ltda, Rio de Janeiro, Brazil

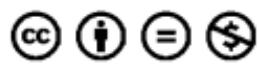




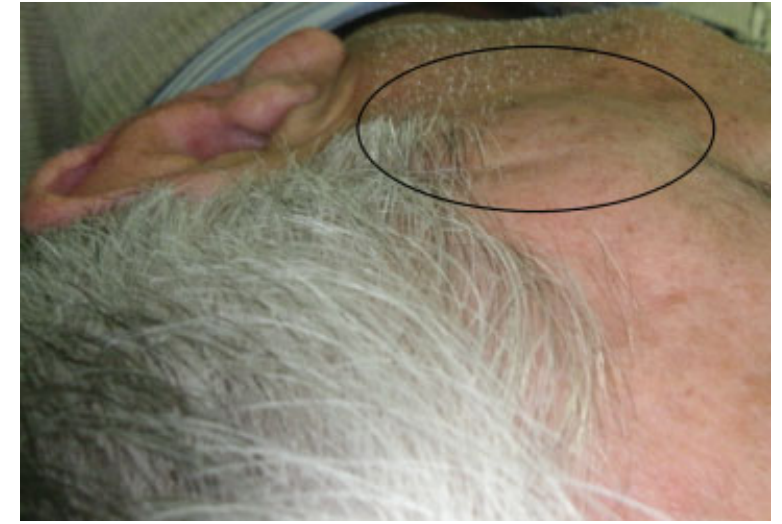

Fig. 1 Increased volume in the left temporal region on physical examination.

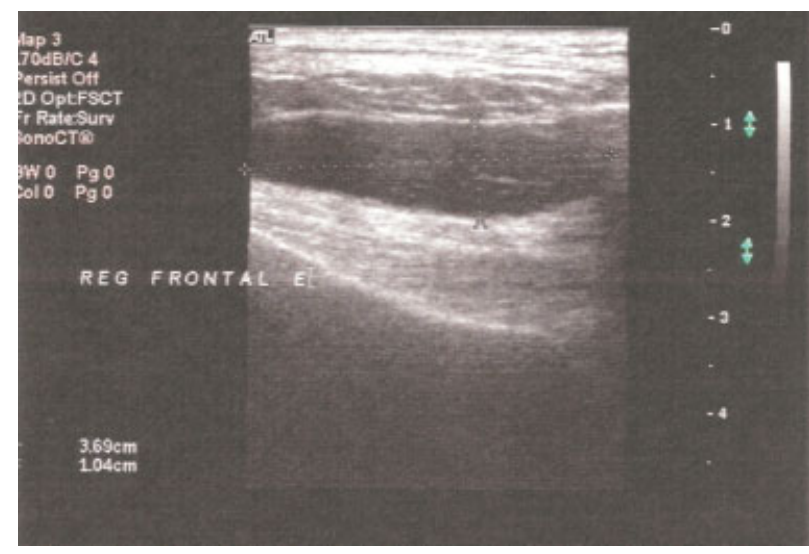

Fig. 2 Ultrasonography revealing a well-defined hypoechoic area, measuring $\sim 3.7 \times 1.0 \mathrm{~cm}$.

radiologists report, "The examined region presented alterations that may correspond to accumulation of subcutaneous tissue." Orthopantographic radiography revealed a change in the morphology of the left mandible condyle (-Fig. 3).

The sum of anamnesis, physical examination, and laboratory tests undoubtedly indicated a gout manifestation in the TMJ. The patient was informed of his condition and referred to a rheumatologic consultation in July 2011. The patient skipped the follow-up consultations.

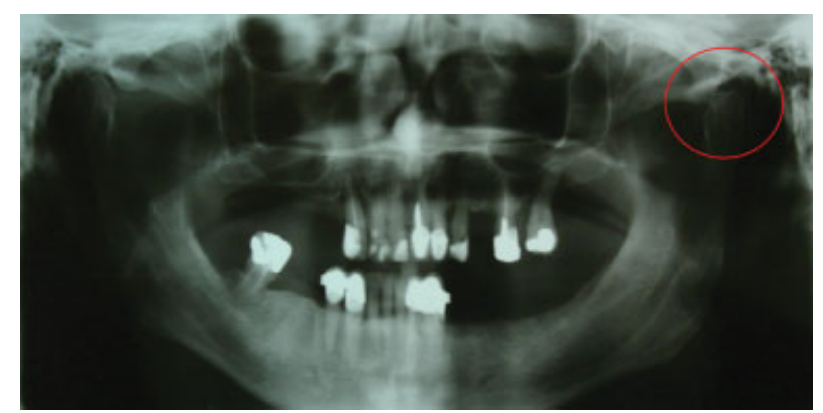

Fig. 3 Orthopantographic radiography revealing a morphologic change of the left mandibular condyle.

\section{Discussion}

Gouty arthritis is a metabolic illness that most often affects middle-aged men and presents with pain, swelling, local sensitivity, and, in the majority of cases, hyperuricemia., ${ }^{2,3}$ Monosodium urate deposits are located mainly in some small joints of the upper and lower limbs., ${ }^{3,4}$ Pain and limitations of mandibular functions may suggest some diagnoses such as arthritis, inflammatory diseases, or tumors of the TMJ. ${ }^{5,6}$ The manifestation of gout should be also included in the diagnoses despite of its rare occurrence. ${ }^{5,6}$

Bhattacharyya et al presented, after a clinical trial, some features of TMJ gout such as limited mouth opening with mandibular deviation and the occurrence of pain, swelling, joint sounds, and impaired movement. ${ }^{5}$ Suba et al also reported decreased mouth opening as a symptom of gout. ${ }^{3}$ Cacioppi et al presented a unique case of malocclusion of the mouth associated with extensive involvement of gout in the whole body as well as symptoms indicating rheumatoid arthritis. ${ }^{1}$ The case presented here showed coincidental features noted by other authors. The patient was a 66 -yearold man complaining of pain and limitations and swelling in the left TMJ region.

Complementary exams can be very helpful when the suspected disease is gout. However, a meticulous anamnesis and a thorough physical examination are paramount for the correct diagnose. ${ }^{1,5,6}$ Because our patient reported a previous blood exam showing hyperuricemia, the clinical investigation was directed to a possible gout manifestation.

According to Suba et $\mathrm{al}^{3}{ }^{3}$ the elevated concentrations of urate in the serum and in the urine or the knowledge of previous episodes of gout can help differentiate the illness from other joint diseases. Because one-third of patients present with hyperuricemia, radiography, computed tomography, and biopsy of the lesions could help confirm suspected cases of gout. Nevertheless, Kleinman and Ewbank asserted that the high levels of uric acid in the bloodstream at the time of the consultation, associated with the remission of the symptoms after drug treatment (NSAIDs, glucocorticoids, and colchicine), suggest gout. ${ }^{6}$ According to them, it also excludes the need for a biopsy. These authors presented three cases of gout diagnosed after careful clinical investigation. The actual case report corroborates Bhattacharyya et $\mathrm{al}^{5}$ and Kleinman and Ewbank, ${ }^{6}$ as it was conducted after a diligent clinical appraisal. We could also study some other exam findings. The ultrasonography presented a well-defined swelling beneath the skin and a left condyle distortion, suggesting gout manifestation in the TMJ.

In the present case report, the sum of anamnesis and the physical and complementary exams pointed to the diagnosis of gout, revealing the sovereign value of the clinical approach.

\section{Conclusion}

Gout in the TMJ, despite being rare, should be included as a differential diagnosis for joint disorders. A previous history of gout with improvement after the use of NSAIDs may favor the diagnosis of gout, leading to the right treatment. The 
complementary exams can be helpful pinpoint TMJ cases of gout and discard some other diseases that normally affect the region.

\section{References}

1 Cacioppi JT, Morrissey JB, Bacon AS. Condyle destruction concomitant with advanced gout and rheumatoid arthritis. Report of a case. Oral Surg Oral Med Oral Pathol 1968;25(6):919-922

2 Schumacher HR, Chen LX. Gota e outras artropatias associadas a cristais. In: Longo DL, Fauci AS, Kasper DD, et al, eds. Medicina interna de harrison. 18 ed. Porto Alegre, Brazil: AMGH; 2013:2837-2842
3 Suba Z, Takács D, Gyulai-Gaál S, et al. Tophaceous gout of the temporomandibular joint: a report of 2 cases. J Oral Maxillofac Surg 2009;67(7):1526-1530

4 Barthélémy I, Karanas Y, Sannajust JP, Emering C, Mondié JM. Gout of the temporomandibular joint: pitfalls in diagnosis. J Craniomaxillofac Surg 2001;29(5):307-310

5 Bhattacharyya I, Chehal H, Gremillion H, Nair M, Odont L. Gout of the temporomandibular joint: a review of the literature. J Am Dent Assoc 2010;141(8):979-985

6 Kleinman HZ, Ewbank RL. Gout of the temporomandibular joint. Report of three cases. Oral Surg Oral Med Oral Pathol 1969;27(2): 281-282 